\title{
Core polarization effects: Oscillator strengths, transition probabilities and radiative lifetimes of levels in Bi IV
}

\author{
C. Moreno-Díaz A. Alonso-Medina
}

\begin{abstract}
A B S T R A C T
In this work a systematic analysis of Bi IV atomic properties is presented. Ab initio relativistic Hartree-Fock calculations in an intermediate coupling (IC) formalism and taking into account Core Polarization Effects (CPE) are used. We use for the IC calculations the standard method of least square fitting of experimental energy levels by means of computer codes from Cowan which was modified in order to include CPE. Our calculations have been made from 17 configurations of Bi IV: $5 d^{10} 6 s^{2}, 5 d^{10} 6 \mathrm{p}^{2}, 5 d^{10} 6 s n s(n=7-10)$ and $5 \mathrm{~d}^{10} 6 \mathrm{snd}(n=6-9)$ for even parity and $5 \mathrm{~d}^{10} 6 \mathrm{snp}(n=6,7), 5 \mathrm{~d}^{10} 6 \mathrm{snf}(n=5,6), 5 \mathrm{~d}^{9} 6 \mathrm{~s}^{2} 6 \mathrm{p}$ and $5 \mathrm{~d}^{9} 6 \mathrm{~s}^{2} \mathrm{nf}$ $(n=5,6)$ for odd parity. Transition probabilities and oscillator strengths for 100 lines and some radiative lifetimes of Bi IV have been calculated. Besides a comparison of our results with the scarce experimental values available in the bibliography are presented. The presence of spectral lines of bismuth in stellar atmospheres has been reported in different stars. This is the motivation by this work can be very interest in astrophysical area. Recently the Bi IV is taking importance because it is considered as the main dopant in the prospective used for the design and synthesis of new scintillators.
\end{abstract}

\section{Introduction}

In the literature, experimental data on the properties of Bi IV have not been found, excepting some values of radiative lifetimes. This is the motivation of present work. Therefore the calculation of the atomic properties of this atomic species is necessary for various applications. Data about atomic properties are relevant not only to spectroscopy; these values are also of interest in a variety of other fields in physics and technology. In astrophysical applications this information can be used to determine elemental abundances from absorption spectra. Although we have not found bibliographical references of the presence of $\mathrm{Bi}$ IV in stellar spectra if there are references of the presence of Bi I, Bi II, and Bi III, as we discussed in our previous work, Colón et al. [1]. Especially the information about oscillator strengths and lifetimes has applications in many scientific fields. Bi IV is an ionic species of high interest in the science of new materials and in the industry. Bi IV can be considered as the main dopant in the prospective used for the design and synthesis of new scintillators, Zorenko et al. [2,3]. This article together with the already made [1], is motivated in part by all this.

The Bi IV together with $\mathrm{Pb}$ III, Tl II and $\mathrm{Hg}$ I form part of the isoelectronic sequence of mercury which has generated studies of fundamental interest in atomic physics since last 30 years. Some atomic parameters of $\mathrm{Pb}$ III were already measured and calculated by various authors. For example Colón et al. [4] have measured 10 lines transition probabilities and others 30 lines transition probabilities were measured by Alonso-Medina [5], using the optical emission from a laser induced plasma. Theoretical calculations were performed by Safranova et al. [6] who have calculated excitation energies, oscillator strengths and several radiative lifetimes, using a hybrid method that combines configuration interaction and linearized coupled-cluster approaches. The transition probabilities of 54 spectral lines of $\mathrm{Pb}$ III, Colón and Alonso-Medina [7], and for the oscillator strengths of 382 spectral lines of $\mathrm{Pb}$ III were performed by Alonso-Medina et al. [8]. In this work a theoretical study, similar to the one previously mentioned [8], is realized obtaining the values in intermediate coupling (IC) and using relativistic Hartree-Fock calculations including core polarization effects. We use for the IC calculations the standard method of leastsquare fitting from experimental energy levels by means of Cowan computer code.
McLay and Crawford [9] did an exhaustive paper where 360 lines of Bi IV were classified, which was collected by Moore, 1958, [10]. Hg I was made by Martin et al. [11]. Chou et al. [12] obtained excitation energies and oscillator strengths from the ${ }^{1} \mathrm{~S}_{0}$ ground state to the first ${ }^{3} \mathrm{P}_{1}^{o}$ and ${ }^{1} \mathrm{P}_{1}^{o}$ exited states of $\mathrm{Hg}$-like ions are calculated by using the multiconfiguration relativistic randomphase approximation including excitation channels from core electrons.

Later, an analysis of transitions from autoionization states of Bi IV was made by Ryabtsev et al. [13]. Later Glowacki and Migdalek [14] realized the relativistic configuration-interaction (CI) method with numerical Dirac-Fock wave functions generated using an ab initio model potential with non-integer outermost core shell occupation number to improve the efficiency of the $\mathrm{CI}$ representation of core-valence electron correlation, this way, the transition energies for $6 \mathrm{~s}^{2}{ }^{1} \mathrm{~S}_{0}-6 \mathrm{~s} 6 \mathrm{p}^{3} \mathrm{P}_{1}, \mathrm{P}_{1}$ transitions in the mercury isoelectronic sequence were obtained. In 2008, the $5 d$ photoabsorption spectra of $\mathrm{Pb}$ III and Bi IV were studied by Banahan et al. [15].

The lifetime of the $5 d^{10} 6 s 6 p^{1} P_{1}$ level was measured by Andersen et al. [16]. In 1988, the lifetimes of nine levels of Bi IV were obtained by Pinnington et al. [17]. Later, lifetime measurements of five levels of Bi IV (including the above mentioned) were made by Ansbacher et al. [18]. These values were obtained using the beam-foil technique and the "correlated analysis of decay curve" (ANDC) method. A summary, of the existing lifetimes for the $6 s^{2}-6 s 6 p$ transitions, has been presented by Curtis et al. [19].

In this work we present transition probabilities and oscillator strengths for 100 spectral lines arising from $5 d^{10} 6$ sns $(n=7,8)$, $5 \mathrm{~d}^{10} 6 \mathrm{p}^{2}, 5 \mathrm{~d}^{10} 6 \operatorname{snp}(\boldsymbol{n}=6,7), 5 \mathrm{~d}^{9} 6 \mathrm{~s}^{2} 6 \mathrm{p}, 5 \mathrm{~d}^{10} 6$ snd $(\boldsymbol{n}=6,7)$ and $5 d^{10} 6 s^{2} 5 f$ configurations of Bi IV. It completes the study of the experimental configurations of the Bi IV, Moore [10]. A comparison between theoretical lifetimes deduced from our calculations and the experimental values available in the bibliography is also presented. As mentioned above, this work has already allowed us to calculate other atomic parameters as the Stark broadening of Bi IV spectral lines are in Colón et al. [1]. Following we shall describe in Section 2 the theoretical considerations, then in Section 3 the discussion and the results. The conclusions are presented in Section 4. 


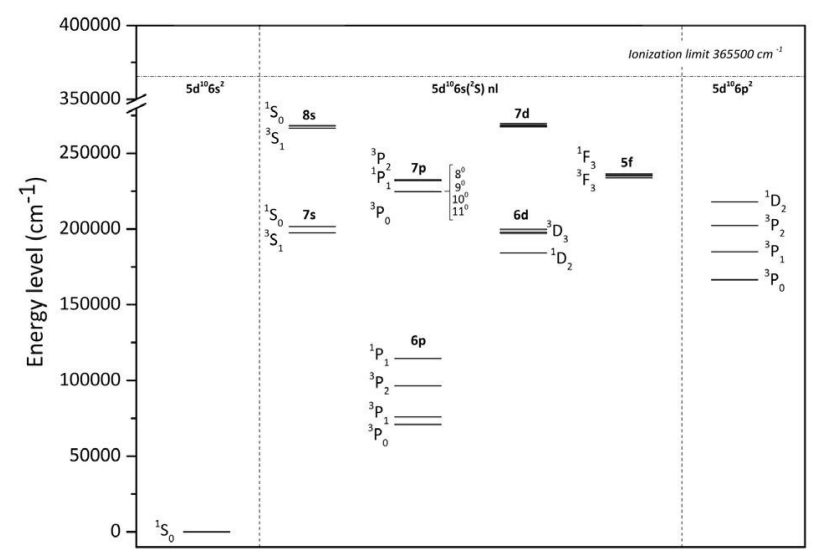

Fig. 1. Energy level diagram of Bi IV (Moore 1958 [10]).

\section{Theoretical calculations}

In order to provide a complete set of transitions probabilities and the corresponding oscillator strengths, relativistic HartreeFock (HFR) method and configuration interaction calculations were made using Cowan's programs, [20]. The basis set used in this work consists of seventeen configurations of Bi IV: $5 d^{10} 6 s^{2}, 5 d^{10} 6 p^{2}$, $5 d^{10} 6$ sns $(n=7-10)$ and $5 d^{10} 6$ snd $(n=6-9)$ for even parity and $5 \mathrm{~d}^{10} 6 \mathrm{snp}(n=6,7), 5 \mathrm{~d}^{10} 6 \mathrm{snf}(n=5,6), 5 \mathrm{~d}^{9} 6 \mathrm{~s} 26 \mathrm{p}$ and $5 \mathrm{~d}^{9} 6 \mathrm{~s} 2 \mathrm{nf}$ ( $n=5,6$ ) for odd parity. For the intermediate coupling (IC) calculations, we used the standard method of least-square fitting of experimental energy levels by means of the computer programs of Cowan [20]. For the calculations we used all the experimental levels $(17+28$ levels) shown in the Moore table, which is based on McLay and Crawford [9] and several autoionization levels of Ryabtsev et al. [13].

Due to the high number of parameters to adjust (that exceeds the number of experimental levels) we have excluded the adjustment process for a certain number of parameters. We take for all the $F^{k}, G^{k}$ and $R^{k}$ integrals not adjusted in the fitting procedure the ab initio HFR values scaled down by a factor of 0.85 (as suggested by Cowan). Our parameters differ slightly from the parameters obtained by Martin et al. [11], which focused on the best possible adjustment of energy levels, as already mentioned in the previous article [1]. For the spin-orbit integrals $\zeta_{n l}$ characterized by small numerical values and not adjusted in the fitting procedures we used the ab initio HFR values without scaling. All the details of the fitting procedure are not given here but can be obtained upon request from the authors. Results of the process for the configuration interactions $5 d^{10} 6 s^{2}, 5 d^{10} 6 p^{2}, 5 d^{10} n s(7,8), 5 d^{10} n p$ $(6,7), 5 d^{10} 5 f, 5 d^{9} 6 s^{2} 6 p, 5 d^{9} 6 s^{2} n f(5,6)$ levels are shown in Tables 1 and 2. In Fig. 1 displays a partial Gotrian scheme of the Bi IV energy levels.

The system considered is complex, with high $Z=83$, where both relativistic and correlation effects must be important. Cowan code has been modified in order to include the core polarization effects (CPE) in a similar way to the works presented by AlonsoMedina et al. [8] and Zanón et al. [21]. These effects are included following the suggestions of Migdalek et al. [22]; they can be written as one-particle, $V_{P 1}$, and two-particle, $V_{P 2}$, potential models,

$V_{P 1}=-\frac{1}{2} \alpha_{d} \sum_{i=1}^{n} \frac{r_{i}^{2}}{\left(r_{i}^{2}+r_{c}^{2}\right)^{3}}$

and

$V_{P 2}=-\alpha_{d} \sum_{i>j}^{n} \frac{\vec{r}_{i} \cdot \vec{r}_{j}}{\left[\left(r_{i}^{2}+r_{c}^{2}\right)\left(r_{j}^{2}+r_{c}^{2}\right)\right]^{3 / 2}}$ where $\alpha_{d}$ is the dipole polarizability of the core and $r_{C}$ is the cutoff radius chosen as a measure of the size of the ionic core. A modification in the radial matrix element can be made in order to take into account the potential change. The matrix element $\left\langle P_{n l}|r| P_{n^{\prime} l}\right\rangle$ is replaced by

$$
\int_{0}^{\infty} P_{n l} r\left(1-\frac{\alpha_{d}}{r^{2}+r_{c}^{2}}\right)^{3 / 2} P_{n^{\prime} l^{\prime}} d r-\frac{\alpha_{d}}{r_{c}^{3}} \int_{0}^{r_{c}} P_{n l}(r) r P_{n^{\prime} l^{\prime}}(r) d r
$$

where the core penetration term suggested by Hameed [23] has also been included. For the dipole polarizability and the cut-off radius we use the values, $\alpha_{d}=3.04$ (in atomic units, a.u.) and $r_{c}=1.201$ (in a.u.), computed by Migdalek and Baylis [24]. In this way we obtained the LS composition of each level and the degree of configuration mixing considering their interactions. For the HFR calculations, the Cowan code provides the radial parts for determining the transition probabilities and initial estimation of the parameters for the IC fittings. The wave functions obtained in this description have been used in this work to obtain the matrix elements and the transition probabilities reported. The transition probabilities and the oscillator strengths are obtained from the matrix elements by using the standard expressions of Martin and Wiese [25]:

$\begin{aligned} f_{j i} & =1.4992 \cdot 10^{16} \lambda^{2} \frac{g_{i}}{g_{j}} A_{i j} \\ A_{i j} & =\frac{2 \pi e^{2}}{m_{e} c \varepsilon_{0} \lambda^{2}} \frac{g_{j}}{g_{i}} f_{j i}=\frac{16 \pi^{3}}{3 h \varepsilon_{0} \lambda^{2}}\left|\left\langle P_{i}|\vec{r}| P_{j}\right\rangle\right|^{2}\end{aligned}$

where $f_{j i}$ and $A_{i j}$ are the oscillator strength and the transition probability respectively, $\lambda$ is the transition wavelength, $g_{i}$ and $g_{j}$ are the statistical weights, $e$ and $m_{e}$ are the electron charge and electron mass, $c$ is the light speed, $h$ is the Planck constant, and $\left\langle P_{i}|\vec{r}| P_{j}\right\rangle$ is the calculated matrix element including the modification pointed out above. The lifetime of a level is the inverse of the sum of the transition probabilities arising from this level. The Lande factors are calculated using the standard expressions given by Cowan [20].

\section{Discussion and results}

In Table 1 we present the wave functions of level corresponding to $5 \mathrm{~d}^{10} 6 \mathrm{~s}^{2}, 5 \mathrm{~d}^{10} 6 \mathrm{p}^{2}, 5 \mathrm{~d}^{10} \mathrm{~ns}(7,8), 5 \mathrm{~d}^{10} \mathrm{np}(6,7), 5 \mathrm{~d}^{10} 5 \mathrm{f}, 5 \mathrm{~d}^{9} 6 \mathrm{~s}^{2} 6 \mathrm{p}$, $5 d^{9} 6 s^{2} n f(5,6)$ configurations in terms of the (LS) functions, the experimental and theoretical energy values as well as the Lande factors. A comparison shows a good agreement, between our calculated energy levels and the experimental values, [10]. In this paper we present the calculated Lande factors that could not be compared in the absence of experimental data. There are no significant discrepancies, except for the energies of the configurations, $5 d^{10} 6 p^{2}$ even parity and the odd parity, $5 d^{10} 6 s 7 p$ and $5 d^{9} 6 s^{2} 6 p$, which appear to be due to the mix of configurations.

As can be seen in Table 1 , for levels $5 d^{10} 6 s\left({ }^{2} S\right) 7 p^{3} P_{1}^{o}$ and ${ }^{3} \mathrm{P}_{0}^{o}$, this work identifies them with energies $224345 \mathrm{~cm}^{-1}$ and $224731 \mathrm{~cm}^{-1}$ while in Moore tables are not registered. The $13^{\circ}$ and $14^{\circ}$ levels identified by Moore as $5 d^{9} 6 s^{2} 6 p$ with energies $228127 \mathrm{~cm}^{-1}$ and $230637 \mathrm{~cm}^{-1}$ have been identified in our work as a mixture of $5 d^{10} 6 s\left({ }^{2} s\right) 7 p{ }^{1} P_{1}$ or $5 d^{10} 6 s\left({ }^{2} S\right) 7 p^{3} P_{1}$ for the $13^{\circ}$ and for the $14^{\circ}$ as the $5 d^{10} 6 s\left({ }^{2} \mathrm{~S}\right) 7 \mathrm{p}^{3} \mathrm{P}_{2}$. For the Moore's levels $5 \mathrm{~d}^{9} 6 \mathrm{~s}^{2} 6 \mathrm{p} 7^{\circ}, 12^{\circ}$ and $22^{\circ}$ we have assigned the terms $\left({ }^{3} \mathrm{P}_{2}-{ }^{3} \mathrm{D}_{2}\right)$, $\left({ }^{3} \mathrm{~F}_{4}\right)$ and $\left({ }^{1} \mathrm{P}_{1}-{ }^{3} \mathrm{D}_{1}\right)$ respectively. In the $15^{\circ}, 16^{\circ}, 19^{\circ}$ and $21^{\circ}$ levels Moore identifies them as $5 d^{10} 6 s\left({ }^{2} S\right) 7 p$ to the first two and $5 d^{10} 6 s\left({ }^{2} S\right) 5 f$ to the last two. While with these calculations the $15^{\circ}$ is $5 d^{9} 6 s^{2} 6 p\left({ }^{3} p_{1}-{ }^{3} D_{1}\right), 5 d^{9} 6 s^{2} 6 p\left({ }^{1} D_{2}-{ }^{3} D_{2}\right)$ to $16^{\circ}$, the $19^{\circ}$ as $5 d^{9} 6 s^{2} 6 p\left({ }^{3} F 2-{ }^{1} D_{2}\right)$ and $21^{\circ}$ has been identified as $5 d^{9} 6 s^{2} 6 p\left({ }^{3} D_{3}\right.$ $\left.-{ }^{1} \mathrm{~F}_{3}\right)$.

The values found for the different parameters involved in the IC calculations, compared with the HFR values used as a start in 
the fitting process, are shown in Table 2. Despite these differences, the parameters obtained do not deviate much from the ab initio parameters; These maintain their physical meaning, allow the best possible adjustment of calculated energy levels, to existing experimental energy levels in the literature, and provide an appropriate set of transition probabilities, oscillator strengths and radiative lifetime.

3.1. Transitions probabilities, oscillator strengths and radiative lifetimes

Theoretical transition probabilities obtained for 100 lines of $\mathrm{Bi}$ IV with wavelengths in the range from 400 to $6200 \dot{A}$ are presented in Tables 3-6. Showing in the first column the transition, the column two gives the corresponding wavelengths, in the third the relative intensity which is collected in the NIST [26]. In the columns four, five and six we display the transition probabilities, oscillator strength and the radiative lifetimes. All the presented values have been calculated with HFR+CPE and HFR. In addition, noticed in the column of the radiative lifetimes, the values of experimental radiative lifetimes of other authors have been included. The calculations, obtained in this work, with core polarization bring us closer to the experimental values. In general, the variation between the results does not reach $10 \%$, with the exception of the calculated radiative lifetime of $5 d^{10} 6 s\left({ }^{2} s\right) 6 d^{1} D_{2}$ whose value with core polarization distances us from the experimental value and $5 d^{10} 6 s\left({ }^{2} S\right) 7 s^{1} S_{0}$ whose value calculated both with core polarization and without it is 0.29 ns compared to $(0.453 \pm 0.026)$ ns of the experimental value.

\section{Conclusion}

In this paper, we have presented transitions probabilities, oscillator strengths and lifetimes of Bi IV configurations. Core polarization effects are included in our calculations. When taking into account in the calculations the effect of core polarization the results obtained for the radiative lifetime are similar to the experimental ones, with an error of $10 \% \mathrm{~m}$ except in the case of level $5 d^{10} 6 s\left({ }^{2} s\right) 7 s{ }^{1} S_{0}$ whose error is of about $35 \%$. The radiative lifetime of the level $5 d^{10} 6 s\left({ }^{2} S\right) 6 d^{1} D_{2}$ without core polarization is identical to the experimental one. Many of the values calculated in this work are the first presented in the bibliography. A new assignment has been given to some of the levels of Moore's tables.

\section{Acknowledgment}

This work was financially supported by the Spanish Ministry of Science and Technology (DGI project MAT-2015-63974-C4-2-R). 


\section{Explanation of Tables}

Table 1 Energy levels and wave functions calculated in Bi IV.

$\begin{array}{ll}\text { Levels } & \text { Numbers assigned to each level } \\ \text { Energy } & \text { Energy of each level, in } \mathrm{cm}^{-1} \\ \text { Lande Factor } & \text { The Lande Factor calculated in this work } \\ J & \text { The total angular moment } \\ \text { Comp } & \text { LS components of each level }\end{array}$

Table 2

Parameters resulting from the least square fitting of the intermediate coupling calculations.

Configuration The configuration, with $[\mathrm{Xe}] 4 \mathrm{f}^{-1}$ truncated

Parameters Parameters, in $\mathrm{cm}^{-1}$

$E_{a v} \quad$ Energy configuration average

$F^{k}, G^{k} \quad$ Coulomb radial integrals

$\zeta_{n l} \quad$ Sin-orbit energy

Table 3 Wavelength ( $\lambda$ in $\AA$ ), relative intensity $\left(I_{\text {rel }}\right)$, transition probabilities $A_{i j}$ (in $\times 10^{8} s^{-1}$ ), oscillator strengths $(f)$ and radiative lifetime $\tau$ (in ns) of spectral lines arising from $5 d^{10} 6 \operatorname{sns}(n=7,8)$ configurations of Bi IV. Our radiative lifetime $\tau$ results are compared with others authors experimental data from (a) Ansbaher et al. [18] and (b)

Pinnington et al. [17].

First column

Second column

Transition level, the lower and upper levels.

Wavelength, $(\lambda)$ in $\AA$

Third column Relative Intensity $\left(I_{\text {rel }}\right)$

Fourth column Transition probabilities $A_{i j}$ (in $\times 10^{8} \mathrm{~s}^{-1}$ ), calculated with HFR+CPE and HFR.

Fifth column Oscillator strengths calculated with HFR+CPE and HFR

Sixth column $\quad$ Radiative lifetime (in ns) calculated with HFR+CPE, HFR and experimental of other authors.

Table $4 \quad$ Wavelength ( $\lambda$ in $\AA$ ), relative intensity $\left(I_{\text {ret }}\right)$, transition probabilities $A_{i j}$ (in $\times 10^{8} s^{-1}$ ), oscillator strengths $(f)$ and radiative lifetime $\tau$ (in ns) of spectral lines arising from $5 d^{10} 6 p^{2}, 5 d^{10} 6 \operatorname{snp}(n=6,7)$ configurations of Bi IV. Our radiative lifetime $\tau$ results are compared with others authors experimental data from (a) Ansbaher et al. [18], (b) Pinnington et al. [17],(c) Curtis et al. [19] and (d) Andersen et al. [16].

First column Transition level, the lower and upper levels.

Second column Wavelength, $(\lambda)$ in $\AA$

Third column Relative Intensity $\left(I_{\text {rel }}\right)$

Fourth column Transition probabilities $A_{i j}$ (in $\times 10^{8} \mathrm{~s}^{-1}$ ), calculated with HFR+CPE and HFR.

Fifth column Oscillator strengths calculated with HFR+CPE and HFR

Sixth column $\quad$ Radiative lifetime (in ns) calculated with HFR+CPE, HFR and experimental of other authors.

Table 5 Wavelength ( $\lambda$ in $\AA$ ), relative intensity $\left(I_{N I S T}\right.$ ), transition probabilities $A_{i j}$ (in $\times 10^{8} s^{-1}$ ), oscillator strengths (f) and radiative lifetime $\tau$ (in ns) of spectral lines arising from $5 d^{10} 6 \operatorname{snd}(n=6,7)$ configurations of Bi IV. Our radiative lifetime $\tau$ results are compared with others authors experimental data from (a) Ansbaher et al. [18] and (b) Pinnington et al. [17].

First column

Second column

Transition level, the lower and upper levels.

Third column

Wavelength, $(\lambda)$ in $\AA$

Relative Intensity $\left(I_{\text {rel }}\right)$

Transition probabilities $\mathrm{A}_{\mathrm{ij}}$ (in $\times 10^{8} \mathrm{~s}^{-1}$ ) calculated with HFR+CPE and HFR.

Fifth column Oscillator strengths calculated with HFR+CPE and HFR

Sixth column $\quad$ Radiative lifetime (in ns) calculated with HFR+CPE, HFR and experimental of other authors.

Table 6

Wavelength ( $\lambda$ in $\AA$ ), relative intensity $\left(I_{\text {rel }}\right)$, transition probabilities $A_{i j}$ (in $\times 10^{8} s^{-1}$ ), oscillator strengths (f) and radiative lifetime $\tau$ (in ns) of spectral lines arising from $5 d^{10} 6 s 5 f$ configurations of $B i$ IV.

First column

Second column

Third column

Fourth column

Fifth column

Transition level, the lower and upper levels.

Wavelength, $(\lambda)$ in $\AA$

Relative Intensity $\left(I_{\text {rel }}\right)$

Transition probabilities $A_{i j}$ (in $\times 10^{8} \mathrm{~s}^{-1}$ ), calculated with HFR+CPE and HFR.

Sixth column

Oscill ator strengths calculated with HFR+CPE and HFR

Radiative lifetime (in ns) calculated with HFR+CPE, HFR and experimental of other authors. 
Table 1

Energy levels and wave functions calculated in $\mathrm{Bi}$ IV.

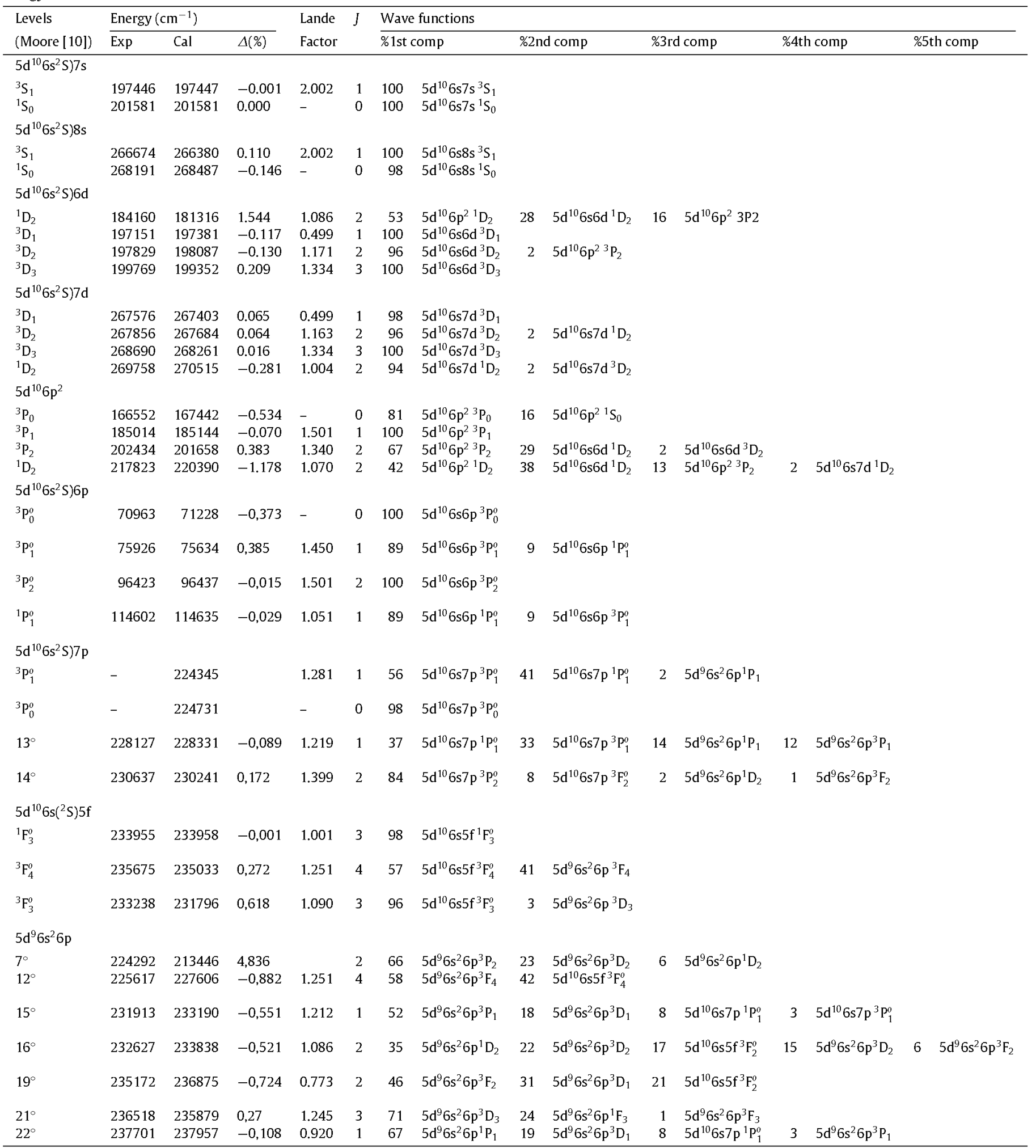


Table 2

Parameters resulting from the least square fitting of the intermediate coupling calculations.

\begin{tabular}{|c|c|c|c|c|c|c|}
\hline Configuration & Parameters $\left(\mathrm{cm}^{-1}\right)$ & & & & & \\
\hline $5 d^{10} 6 s^{2}$ & $\mathrm{E}_{a v}=3.5810$ & & & & & \\
\hline $5 d^{10} 6 p^{2}$ & $\mathrm{E}_{a v}=196.8488$ & (193.954) & $\mathrm{F}^{2}(6 \mathrm{p}, 6 \mathrm{p})=32.44$ & $(37.39)$ & $\zeta_{6 p}=15.6242$ & $(15.4718)$ \\
\hline $5 d^{10} 6 s 7 s$ & $\mathrm{E}_{a v}=198.5149$ & $(192.4578)$ & $\mathrm{G}^{0}(6 \mathrm{~s}, 7 \mathrm{~s})=2.1049$ & $(2.9878)$ & & \\
\hline $5 d^{10} 6 s 8 s$ & $\mathrm{E}_{a v}=266.8741$ & $(260.4406)$ & $\mathrm{G}^{0}(6 \mathrm{~s}, 8 \mathrm{~s})=0.9956$ & & & \\
\hline $5 d^{10} 6 s 6 d$ & $\mathrm{E}_{a v}=199.7863$ & (193.2219) & & & & \\
\hline $5 d^{10} 6 s 7 d$ & $\mathrm{E}_{a v}=268.2897$ & $(261.9683)$ & & & & \\
\hline $5 d^{10} 6 s 6 p$ & $\mathrm{E}_{a v}=99.8726$ & $(89.9895)$ & $G^{1}(6 s, 6 p)=38.3197$ & $(46.5527)$ & $\zeta_{6 p}=16.8724$ & (15.6153) \\
\hline $5 d^{10} 6 s 7 p$ & $\mathrm{E}_{a v}=228.3972$ & $(223.9513)$ & $G^{1}(6 s, 7 p)=0.000$ & $(5.7208)$ & $\zeta_{7 p}=3.5518$ & $(4.7777)$ \\
\hline $5 d^{10} 6 s 5 f$ & $\mathrm{E}_{a v}=232.4200$ & $(228.9248)$ & $G^{3}(6 s, 5 f)=7.7288$ & $(16.0186)$ & $\zeta_{5 f}=0.0800$ & \\
\hline $5 d^{9} 6 s^{2} 6 p$ & $\mathrm{E}_{a v}=235.1752$ & $(232.8618)$ & $\begin{array}{l}\mathrm{F}^{2}(5 \mathrm{~d}, 6 \mathrm{p})=21.5807 \\
\mathrm{G}^{1}(5 \mathrm{~d}, 6 \mathrm{p})=5.1544 \\
\mathrm{G}^{3}(5 \mathrm{~d}, 6 \mathrm{p})=8.8610\end{array}$ & $\begin{array}{l}(27.9711) \\
(9.9404)\end{array}$ & $\begin{array}{l}\zeta_{5 d}=8.2057 \\
\zeta_{6 p}=11.9717\end{array}$ & $\begin{array}{l}(9.9623) \\
(17.4360)\end{array}$ \\
\hline $5 d^{9} 6 s^{2} 5 f$ & $\mathrm{E}_{a v}=378.1588$ & $(382.1734)$ & & & & \\
\hline $5 d^{9} 6 s^{2} 6 f$ & $\mathrm{E}_{a v}=422.6762$ & $(429.9606)$ & & & & \\
\hline
\end{tabular}


Table 3

Wavelength $\left(\lambda\right.$ in $\AA$ ), relative intensity $\left(I_{r e l}\right)$, transition probabilities $A_{i j}$ (in $\times 10^{8} \mathrm{~s}^{-1}$ ), oscillator strengths $(f)$ and radiative lifetime $\tau$ (in ns) of spectral lines arising from $5 \mathrm{~d}^{10} 6 \mathrm{sns}(n=7,8)$ configurations of Bi IV.

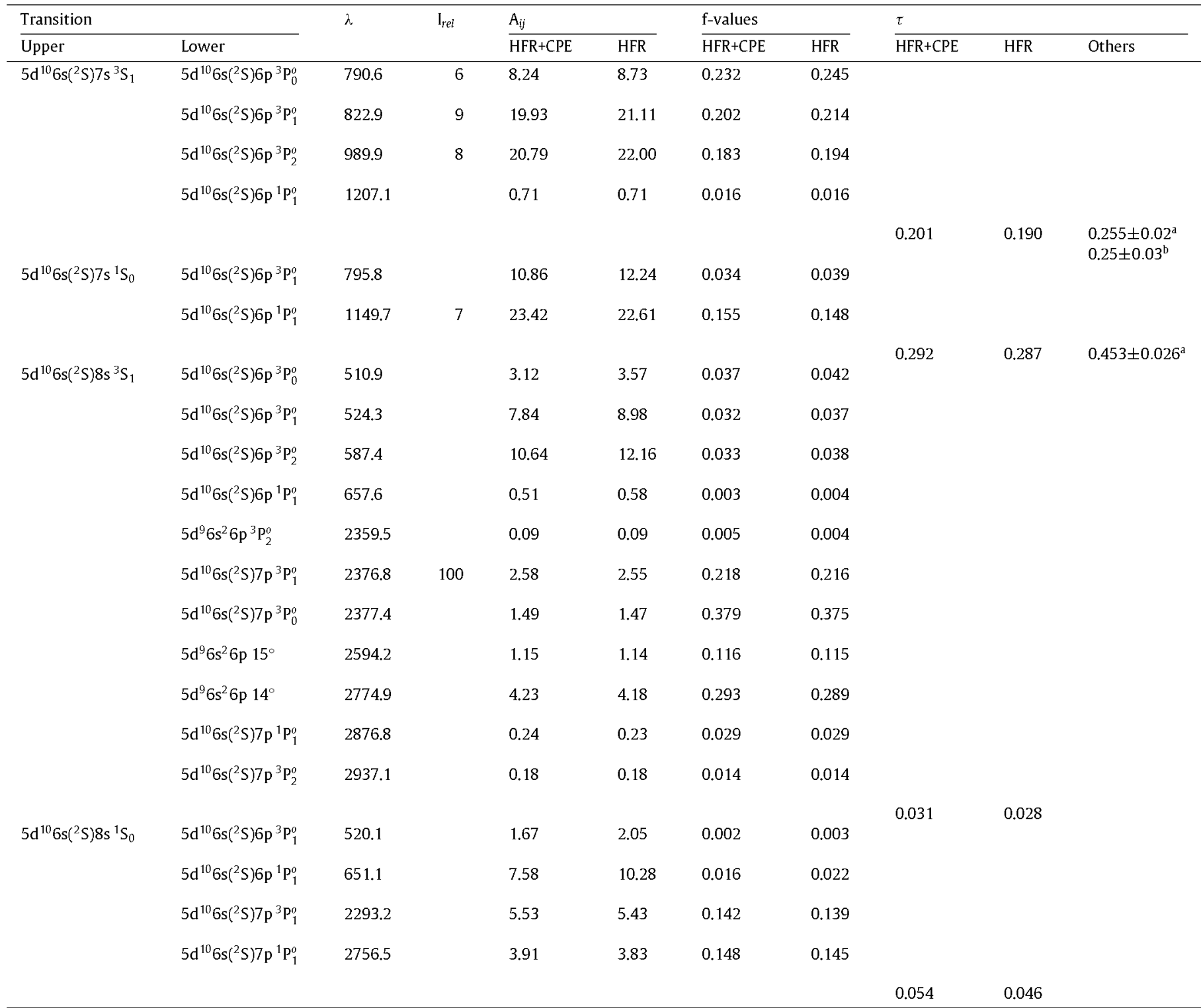

aur radiative lifetime $\tau$ results are compared with others authors experimental data from Ansbaher et al. [18].

${ }^{\mathrm{b}}$ Our radiative lifetime $\tau$ results are compared with others authors experimental data from Pinnington et al. [17]. 
Table 4

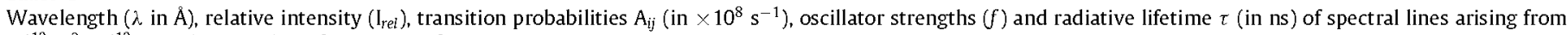
$5 \mathrm{~d}^{10} 6 \mathrm{p}^{2}, 5 \mathrm{~d}^{10} 6 \mathrm{snp}(n=6,7)$ configurations of Bi IV.

\begin{tabular}{|c|c|c|c|c|c|c|c|c|c|c|}
\hline \multicolumn{2}{|l|}{ Transition } & \multirow[t]{2}{*}{$\lambda$} & \multirow[t]{2}{*}{$\mathrm{I}_{\text {rel }}$} & \multicolumn{2}{|l|}{$\mathrm{A}_{\mathrm{ij}}$} & \multicolumn{2}{|l|}{ f-values } & \multicolumn{3}{|l|}{$\tau$} \\
\hline Upper & Lower & & & $\overline{\mathrm{HFR}+\mathrm{CPE}}$ & HFR & $\overline{\mathrm{HFR}+\mathrm{CPE}}$ & HFR & HFR+CPE & HFR & Others \\
\hline \multirow[t]{3}{*}{$5 d^{10} 6 p^{2}{ }^{3} P_{0}$} & $5 d^{10} 6 s\left({ }^{2} s\right) 6 p^{3} P_{1}^{0}$ & 1103.4 & 24 & 21.88 & 48.49 & 0.133 & 0.295 & & & \\
\hline & $5 d^{10} 6 s\left({ }^{2} s\right) 6 p^{1} p_{1}^{0}$ & 1924.9 & & 0.096 & 0.24 & 0.002 & 0.004 & & & \\
\hline & & & & & & & & 0.455 & 0.205 & $0.38 \pm 0.05^{b}$ \\
\hline \multirow[t]{5}{*}{$5 d^{10} 6 p^{2}{ }^{3} P_{1}$} & $5 d^{10} 6 s\left({ }^{2} s\right) 6 p^{3} p_{0}^{0}$ & 876.8 & 8 & 13.59 & 29.90 & 0.469 & 1.033 & & & \\
\hline & $\left.5 d^{10} 6 s^{2} s\right) 6 p^{3} p_{1}^{0}$ & 916.7 & 9 & 8.13 & 17.88 & 0.102 & 0.225 & & & \\
\hline & $5 d^{10} 6 s\left({ }^{2} s\right) 6 p^{3} P_{2}^{0}$ & 1128.8 & 7 & 8.01 & 17.63 & 0.092 & 0.202 & & & \\
\hline & $5 d^{10} 6 s\left({ }^{2} s\right) 6 p{ }^{1} p_{1}^{2}$ & 1420.2 & & 0.247 & 0.543 & 0.007 & 0.016 & & & \\
\hline & & & & & & & & 0.334 & 0.152 & $0.30 \pm 0.03^{b}$ \\
\hline \multirow{4}{*}{$5 d^{10} 6 p^{2}{ }^{3} P_{2}$} & $5 d^{10} 6 s\left({ }^{2} s\right) 6 p^{3} P_{1}^{0}$ & 790.5 & 6 & 10.44 & 21.44 & 0.163 & 0.334 & & & \\
\hline & $\left.5 d^{10} 6 s^{2} s\right) 6 p^{3} p_{2}^{0}$ & 943.3 & 15 & 20.12 & 42.56 & 0.268 & 0.577 & & & \\
\hline & $\left.5 d^{10} 6 s^{2} s\right) 6 p^{1} p_{1}^{0}$ & 1138.5 & 6 & 3.89 & 4.40 & 0.126 & 0.143 & & & \\
\hline & & & & & & & & 0.290 & 0.146 & $0.31 \pm 0.03^{\mathrm{b}}$ \\
\hline \multirow[t]{4}{*}{$5 d^{10} 6 p^{2}{ }^{1} D_{2}$} & $5 d^{10} 6 s\left({ }^{2} S\right) 6 p^{3} P_{1}^{o}$ & 704.7 & & 4.75 & 6.95 & 0.059 & 0.083 & & & \\
\hline & $5 d^{10} 6 s\left({ }^{2} s\right) 6 p^{3} p_{2}^{0}$ & 823.7 & & 5.01 & 11.23 & 0.051 & 0.114 & & & \\
\hline & $5 d^{10} 6 s\left({ }^{2} s\right) 6 p^{1} P_{1}^{o}$ & 968.8 & 8 & 62.38 & 109.14 & 1.463 & 2.559 & & & \\
\hline & & & & & & & & 0.138 & 0.078 & $0.173 \pm 0.024^{\mathrm{a}}$ \\
\hline \multirow{2}{*}{$5 d^{10} 6 s\left({ }^{2} s\right) 6 p^{3} p_{1}^{0}$} & $5 d^{10} 6 s^{2}{ }^{1} S_{0}$ & 1317.1 & 60 & 0.86 & 1.79 & 0.067 & 0.140 & & & \\
\hline & & & & & & & & 11.578 & 5.559 & $\begin{array}{l}8.0 \pm 0.6^{b} \\
9.1 \pm 0.7^{c}\end{array}$ \\
\hline \multirow{4}{*}{$5 \mathrm{~d}^{10} 6 \mathrm{~s}\left({ }^{2} \mathrm{~s}\right) 6 \mathrm{p}{ }^{1} \mathrm{P}_{1}^{0}$} & $5 d^{10} 6 s^{2}{ }^{1} s_{0}$ & 872.6 & 15 & 27.62 & 56.50 & 0.946 & 1.935 & & & \\
\hline & & & & & & & & 0.362 & 0.177 & $0.243 \pm 0.013^{\mathrm{a}}$ \\
\hline & & & & & & & & & & $0.229 \pm 0.014^{b}$ \\
\hline & & & & & & & & & & $0.39 \pm 0.08^{\mathrm{d}}$ \\
\hline \multirow{3}{*}{$5 d^{10} 6 s\left({ }^{2} s\right) 7 p{ }^{3} P_{0}^{0}$} & $5 d^{10} 6 s\left({ }^{2} s\right) 6 d^{3} D_{1}$ & 3645.2 & 100 & 2.01 & 2.18 & 0.134 & 0.145 & & & \\
\hline & $5 d^{10} 6 s\left({ }^{2} s\right) 7 s^{3} s_{1}$ & 3684.8 & 100 & 2.09 & 2.39 & 0.142 & 0.162 & & & \\
\hline & & & & & & & & 2.439 & 2.188 & \\
\hline \multirow{7}{*}{$5 d^{10} 6 s\left({ }^{2} s\right) 7 p^{3} P_{1}^{0}$} & $5 d^{10} 6 s^{2}{ }^{1} S_{0}$ & 445.2 & & 4.69 & 4.27 & 0.042 & 0.038 & & & \\
\hline & $5 d^{10} 6 s\left({ }^{2} s\right) 6 d^{1} D_{2}$ & 2472.7 & & 0.74 & 0.81 & 0.041 & 0.044 & & & \\
\hline & $5 d^{10} 6 s\left({ }^{2} s\right) 6 d^{3} D_{1}$ & 3643.0 & 100 & 0.27 & 0.29 & 0.054 & 0.059 & & & \\
\hline & $\left.5 d^{10} 6 s^{2} s\right) 7 s^{3} s_{1}$ & 3682.6 & 100 & 1.13 & 1.29 & 0.229 & 0.262 & & & \\
\hline & $5 d^{10} 6 s\left({ }^{2} s\right) 6 d^{3} D_{2}$ & 3735.3 & 100 & 0.88 & 0.95 & 0.110 & 0.119 & & & \\
\hline & $\left.5 d^{10} 6 s^{2} s\right) 7 s^{1} S_{0}$ & 4344.1 & 30 & 0.53 & 0.60 & 0.449 & 0.513 & & & \\
\hline & & & & & & & & 1.214 & 1.218 & \\
\hline \multirow{6}{*}{$5 \mathrm{~d}^{10} 6 \mathrm{~s}\left({ }^{2} \mathrm{~s}\right) 7 \mathrm{p}{ }^{1} \mathrm{P}_{1}^{0}$} & $5 d^{10} 6 s\left({ }^{2} s\right) 6 d^{1} D_{2}$ & 2094.1 & 30 & 0.28 & 0.32 & 0.011 & 0.013 & & & \\
\hline & $5 d^{10} 6 s\left({ }^{2} s\right) 6 d^{3} D_{1}$ & 2876.7 & & 0.09 & 0.10 & 0.012 & 0.013 & & & \\
\hline & $5 d^{10} 6 s\left({ }^{2} s\right) 6 d^{3} D_{2}$ & 2933.9 & 100 & 0.21 & 0.22 & 0.016 & 0.017 & & & \\
\hline & $5 d^{10} 6 s\left({ }^{2} s\right) 7 s^{3} s_{1}$ & 2901.3 & & 0.44 & 0.51 & 0.056 & 0.064 & & & \\
\hline & $5 d^{10} 6 s\left({ }^{2} s\right) 7 s^{1} s_{0}$ & 3296.9 & & 0.37 & 0.43 & 0.181 & 0.211 & & & \\
\hline & & & & & & & & 7.194 & 6.329 & \\
\hline \multirow[t]{3}{*}{$5 d^{10} 6 s\left({ }^{2} s\right) 7 p^{3} p_{2}^{0}$} & $5 d^{10} 6 s\left({ }^{2} s\right) 7 s^{3} s_{1}$ & 2842.4 & 100 & 0.23 & 0.26 & 0.047 & 0.053 & & & \\
\hline & $5 d^{10} 6 s\left({ }^{2} s\right) 6 d^{3} D_{3}$ & 3043.4 & 100 & 0.18 & 0.20 & 0.018 & 0.019 & & & \\
\hline & & & & & & & & 24.390 & 21.739 & \\
\hline \multirow[t]{3}{*}{$5 d^{9} 6 s^{2} 6 p 14^{\circ}$} & $5 d^{10} 6 s\left({ }^{2} s\right) 7 s^{3} S_{1}$ & 3012.9 & 100 & 3.14 & 3.59 & 0.712 & 0.815 & & & \\
\hline & $5 d^{10} 6 s\left({ }^{2} S\right) 6 d^{3} D_{2}$ & 3239.6 & 100 & 2.02 & 2.18 & 0.227 & 0.245 & & & \\
\hline & & & & & & & & 1.938 & 1.733 & \\
\hline $5 d^{9} 6 s^{2} 6 p 12^{\circ}$ & $5 d^{10} 6 s\left({ }^{2} s\right) 6 d^{3} D_{3}$ & 3868.7 & 100 & 0.68 & 0.92 & 0.196 & 0.266 & 14.706 & 10.869 & \\
\hline
\end{tabular}

${ }^{a}$ Our radiative lifetime $\tau$ results are compared with others authors experimental data from Ansbaher et al. [18].

${ }^{b}$ Our radiative lifetime $\tau$ results are compared with others authors experimental data from Pinnington et al. [17].

${ }^{c}$ Our radiative lifetime $\tau$ results are compared with others authors experimental data from Curtis et al. [19].

${ }^{d}$ Our radiative lifetime $\tau$ results are compared with others authors experimental data from Andersen et al. [16]. 
Table 5

Wavelength $\left(\lambda\right.$ in $\AA$ ), relative intensity $\left(I_{r e l}\right)$, transition probabilities $A_{i j}$ (in $\times 10^{8} \mathrm{~s}^{-1}$ ), oscillator strengths $(f)$ and radiative lifetime $\tau$ (in ns) of spectral lines arising from $5 \mathrm{~d}^{10}$ 6snd $(n=6,7)$ configurations of Bi IV.

\begin{tabular}{|c|c|c|c|c|c|c|c|c|c|c|}
\hline \multicolumn{2}{|l|}{ Transition } & \multirow[t]{2}{*}{$\lambda$} & \multirow[t]{2}{*}{$\mathrm{I}_{\text {rel }}$} & \multicolumn{2}{|l|}{$A_{i j}$} & \multicolumn{2}{|l|}{ f-values } & \multicolumn{3}{|l|}{$\tau$} \\
\hline Upper & Lower & & & HFR+CPE & HFR & HFR+CPE & HFR & $\overline{\mathrm{HFR}+\mathrm{CPE}}$ & HFR & Others \\
\hline \multirow[t]{4}{*}{$5 d^{10} 6 s\left({ }^{2} S\right) 6 d^{1} D_{2}$} & $5 d^{10} 6 s\left({ }^{2} s\right) 6 p^{3} P_{1}^{o}$ & 923.9 & 12 & 1.27 & 4.19 & 0.027 & 0.089 & & & \\
\hline & $5 d^{10} 6 s\left({ }^{2} S\right) 6 p^{3} P_{2}^{0}$ & 1139.7 & 6 & 2.37 & 5.14 & 0.046 & 0.100 & & & \\
\hline & $5 d^{10} 6 s\left({ }^{2} S\right) 6 p^{1} p_{1}^{2}$ & 1437.7 & & 0.0038 & 0.34 & 0.0002 & 0.017 & & & \\
\hline & & & & & & & & 2.744 & 1.034 & $1.02 \pm 0.11^{\mathrm{d}}$ \\
\hline \multirow[t]{5}{*}{$5 d^{10} 6 s\left({ }^{2} S\right) 6 d^{3} D_{1}$} & $5 \mathrm{~d}^{10} 6 \mathrm{~s}\left({ }^{2} \mathrm{~S}\right) 6 \mathrm{p}^{3} \mathrm{P}_{0}^{0}$ & 792.5 & 8 & 36.43 & 52.57 & 1.029 & 1.485 & & & \\
\hline & $5 d^{10} 6 s\left({ }^{2} s\right) 6 p^{3} p_{1}^{0}$ & 824.9 & 12 & 22.04 & 31.80 & 0.225 & 0.324 & & & \\
\hline & $5 d^{10} 6 s\left({ }^{2} s\right) 6 p^{3} p_{2}^{o}$ & 992.8 & & 0.93 & 1.34 & 0.008 & 0.012 & & & \\
\hline & $5 d^{10} 6 s\left({ }^{2} S\right) 6 p{ }^{1} P_{1}^{o}$ & 1211.4 & & 0.77 & 1.11 & 0.017 & 0.024 & & & \\
\hline & & & & & & & & 0.166 & 0.115 & $0.23 \pm 0.03^{b}$ \\
\hline \multirow[t]{4}{*}{$5 d^{10} 6 s\left({ }^{2} S\right) 6 d^{3} D_{2}$} & $5 d^{10} 6 s\left({ }^{2} S\right) 6 p^{3} p_{1}^{o}$ & 820.3 & 10 & 49.02 & 73.06 & 0.824 & 1.228 & & & \\
\hline & $5 \mathrm{~d}^{10} 6 \mathrm{~s}\left({ }^{2} \mathrm{~S}\right) 6 \mathrm{p}^{3} \mathrm{P}_{2}^{0}$ & 986.1 & & 5.21 & 6.62 & 0.076 & 0.097 & & & \\
\hline & $5 d^{10} 6 s\left({ }^{2} S\right) 6 p^{1} P_{1}^{0}$ & 1201.5 & & 0.58 & 0.84 & 0.021 & 0.030 & & & \\
\hline & & & & & & & & 0.182 & 0.124 & $0.18 \pm 0.03^{b}$ \\
\hline \multirow[t]{2}{*}{$5 d^{10} 6 s\left({ }^{2} S\right) 6 d^{3} D_{3}$} & $5 d^{10} 6 s\left({ }^{2} S\right) 6 p^{3} P_{2}^{0}$ & 967.6 & 9 & 35.33 & 51.01 & 0.694 & 1.003 & & & \\
\hline & & & & & & & & 0.283 & 0.196 & $0.25 \pm 0.03^{b}$ \\
\hline \multirow[t]{11}{*}{$5 d^{10} 6 s\left({ }^{2} S\right) 7 d^{3} D_{1}$} & $5 d^{10} 6 s\left({ }^{2} S\right) 6 p^{3} P_{0}^{o}$ & 508.6 & & 4.78 & 10.89 & 0.056 & 0.128 & & & \\
\hline & $5 d^{10} 6 s\left({ }^{2} S\right) 6 p^{3} p_{1}^{o}$ & 521.8 & & 3.01 & 6.86 & 0.012 & 0.028 & & & \\
\hline & $5 d^{10} 6 s\left({ }^{2} S\right) 6 p^{3} P_{2}^{o}$ & 584.3 & & 0.17 & 0.37 & 0.0005 & 0.001 & & & \\
\hline & $5 d^{10} 6 s\left({ }^{2} S\right) 6 p^{1} p_{1}^{0}$ & 653.7 & & 0.21 & 0.45 & 0.001 & 0.003 & & & \\
\hline & $5 d^{10} 6 s\left({ }^{2} s\right) 7 p^{3} p_{0}^{0}$ & 2326.1 & 100 & 6.03 & 6.41 & 1.467 & 1.561 & & & \\
\hline & $5 d^{10} 6 s\left({ }^{2} S\right) 7 p^{3} P_{1}^{o}$ & 2326.9 & 100 & 2.61 & 2.77 & 0.212 & 0.225 & & & \\
\hline & $5 d^{9} 6 s^{2} 6 p 13^{\circ}$ & 2534.9 & & 1.18 & 1.25 & 0.113 & 0.120 & & & \\
\hline & $5 d^{10} 6 s\left({ }^{2} S\right) 7 p{ }^{1} P_{1}^{o}$ & 2804.0 & & 0.24 & 0.26 & 0.028 & 0.030 & & & \\
\hline & $5 d^{10} 6 s\left({ }^{2} S\right) 7 p^{3} p_{2}^{0}$ & 2861.1 & & 0.31 & 0.27 & 0.023 & 0.019 & & & \\
\hline & $5 \mathrm{~d}^{10} 6 \mathrm{~s}\left({ }^{2} \mathrm{~S}\right) 5 \mathrm{f}^{3} \mathrm{~F}_{2}^{0}$ & 3086.0 & & 0.32 & 0.28 & 0.027 & 0.024 & & & \\
\hline & & & & & & & & 0.530 & 0.335 & \\
\hline \multirow[t]{8}{*}{$5 d^{10} 6 s\left({ }^{2} S\right) 7 d^{3} D_{2}$} & $5 d^{10} 6 s\left({ }^{2} S\right) 6 p^{3} P_{1}^{0}$ & 521.0 & & 6.12 & 14.07 & 0.042 & 0.095 & & & \\
\hline & $5 d^{10} 6 s\left({ }^{2} S\right) 6 p^{3} p_{2}^{0}$ & 583.3 & & 1.55 & 3.48 & 0.008 & 0.018 & & & \\
\hline & $5 d^{10} 6 s\left({ }^{2} S\right) 7 p^{3} p_{1}^{o}$ & 2311.8 & 100 & 6.05 & 6.44 & 0.808 & 0.859 & & & \\
\hline & $5 d^{9} 6 s^{2} 6 p 13^{\circ}$ & 2517.1 & & 1.45 & 1.55 & 0.230 & 0.245 & & & \\
\hline & $5 d^{9} 6 s^{2} 6 p 14$ & 2686.8 & & 1.84 & 1.91 & 0.199 & 0.207 & & & \\
\hline & $5 d^{10} 6 s\left({ }^{2} S\right) 7 p^{1} P_{1}^{o}$ & 2782.2 & & 0.29 & 0.32 & 0.058 & 0.062 & & & \\
\hline & $5 d^{9} 6 s^{2} 6 p 17^{\circ}$ & 2888.7 & & 1.04 & 0.85 & 0.093 & 0.076 & & & \\
\hline & & & & & & & & 0.545 & 0.349 & \\
\hline \multirow[t]{7}{*}{$5 d^{10} 6 s\left({ }^{2} S\right) 7 d^{3} D_{3}$} & $5 d^{10} 6 s\left({ }^{2} S\right) 6 p^{3} P_{2}^{0}$ & 580.5 & & 6.09 & 13.65 & 0.043 & 0.097 & & & \\
\hline & $5 d^{9} 6 s^{2} 6 p 7^{\circ}$ & 2252.4 & & 0.14 & 0.15 & 0.015 & 0.016 & & & \\
\hline & $5 d^{9} 6 s^{2} 6 p 12^{\circ}$ & 2321.6 & & 0.39 & 0.29 & 0.025 & 0.018 & & & \\
\hline & $5 d^{9} 6 s^{2} 6 p 14^{\circ}$ & 2627.9 & & 6.58 & 7.01 & 0.954 & 1.020 & & & \\
\hline & $5 d^{10} 6 s\left({ }^{2} S\right) 7 p^{3} P_{2}^{o}$ & 2772.9 & 100 & 0.31 & 0.33 & 0.051 & 0.054 & & & \\
\hline & $5 d^{10} 6 s\left({ }^{2} S\right) 5 f^{3} F_{4}^{0^{2}}$ & 3027.3 & & 0.77 & 0.66 & 0.082 & 0.070 & & & \\
\hline & & & & & & & & 0.700 & 0.453 & \\
\hline \multirow[t]{8}{*}{$5 d^{10} 6 s\left({ }^{2} S\right) 7 d^{1} D_{2}$} & $5 d^{10} 6 s\left({ }^{2} S\right) 6 p^{3} P_{1}^{o}$ & 515.9 & & 0.55 & 1.54 & 0.004 & 0.010 & & & \\
\hline & $5 \mathrm{~d}^{10} 6 \mathrm{~s}\left({ }^{2} \mathrm{~S}\right) 6 \mathrm{p}{ }^{1} \mathrm{P}_{1}^{0}$ & 644.5 & & 6.13 & 16.83 & 0.064 & 0.175 & & & \\
\hline & $5 d^{10} 6 s\left({ }^{2} S\right) 7 p^{3} p_{1}^{0}$ & 2214.5 & & 3.36 & 3.59 & 0.412 & 0.439 & & & \\
\hline & $5 d^{9} 6 s^{2} 6 p 13^{\circ}$ & 2402.1 & & 4.38 & 4.64 & 0.631 & 0.669 & & & \\
\hline & $5 d^{10} 6 s\left({ }^{2} s\right) 7 p{ }^{1} P_{1}^{o}$ & 2642.4 & & 0.92 & 0.99 & 0.160 & 0.173 & & & \\
\hline & $5 d^{10} 6 s\left({ }^{2} S\right) 5 f^{3} F_{3}^{0}$ & 2793.1 & & 1.67 & 1.39 & 0.139 & 0.116 & & & \\
\hline & $5 d^{9} 6 s^{2} 6 p 22^{\circ}$ & 3119.4 & & 0.38 & 0.41 & 0.092 & 0.101 & & & \\
\hline & & & & & & & & 0.575 & 0.340 & \\
\hline
\end{tabular}

${ }^{a}$ Our radiative lifetime $\tau$ results are compared with others authors experimental data from Ansbaher et al. [18].

${ }^{\mathrm{b}}$ Our radiative lifetime $\tau$ results are compared with others authors experimental data from Pinnington et al. [17].

Table 6

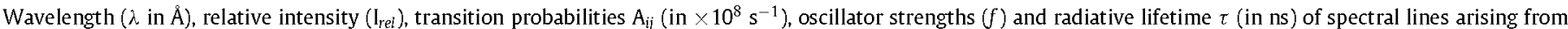
$5 d^{10} 6 s 5 f$ configurations of Bi IV.

\begin{tabular}{|c|c|c|c|c|c|c|c|c|c|c|}
\hline \multicolumn{2}{|l|}{ Transition } & \multirow[t]{2}{*}{$\lambda$} & \multirow[t]{2}{*}{$\mathrm{I}_{\text {rel }}$} & \multicolumn{2}{|l|}{$A_{i j}$} & \multicolumn{2}{|l|}{ f-values } & \multicolumn{3}{|l|}{$\tau$} \\
\hline Uррег & Lower & & & $\mathrm{HFR}+\mathrm{CPE}$ & HFR & $\mathrm{HFR}+\mathrm{CPE}$ & HFR & $\mathrm{HFR}+\mathrm{CPE}$ & HFR & Others \\
\hline $5 d^{10} 6 s\left({ }^{2} S\right) 5 f^{3} F_{3}^{0}$ & $\begin{array}{l}5 d^{10} 6 s\left({ }^{2} S\right) 6 d^{1} D_{2} \\
5 d^{10} 6 p^{2}{ }^{3} P_{2} \\
5 d^{10} 6 p^{2}{ }^{1} D_{2}\end{array}$ & $\begin{array}{l}2008.3 \\
3172.5 \\
6198.9\end{array}$ & & $\begin{array}{l}2.89 \\
0.84 \\
0.10\end{array}$ & $\begin{array}{l}3.94 \\
1.10 \\
0.13\end{array}$ & $\begin{array}{l}0.245 \\
0.177 \\
0.082\end{array}$ & $\begin{array}{l}0.334 \\
0.233 \\
0.103\end{array}$ & 2611 & 1934 & \\
\hline $5 d^{10} 6 s\left({ }^{2} S\right) 5 f^{3} F_{2}^{0}$ & $\begin{array}{l}5 d^{10} 6 s\left({ }^{2} S\right) 6 d^{3} D_{1} \\
5 d^{10} 6 s\left({ }^{2} S\right) 6 d^{3} D_{2}\end{array}$ & $\begin{array}{l}2630.1 \\
2677.9\end{array}$ & $\begin{array}{l}100 \\
100\end{array}$ & $\begin{array}{l}0.91 \\
0.17\end{array}$ & $\begin{array}{l}1.21 \\
0.22\end{array}$ & $\begin{array}{l}0.158 \\
0.018\end{array}$ & $\begin{array}{l}0.209 \\
0.024\end{array}$ & 9259 & 6.993 & \\
\hline $5 d^{10} 6 s\left({ }^{2} S\right) 5 f^{3} F_{4}^{o}$ & $5 d^{10} 6 s\left({ }^{2} S\right) 6 d^{3} D_{3}$ & 2786.5 & 100 & 2.03 & 2.69 & 0.303 & 0.403 & 4.926 & 3.718 & \\
\hline
\end{tabular}

